\title{
MJMR CASE SERIES OF NEOVASCULAR GLAUCOMA:EARLY LIMITEDTRANS- SCLERAL CYTOPHOTOCOAGULATIONS (TSCPC) AS AN OPTIONS FOR PREPARATION OF FILTERING SURGERY IN NVG PATIENTS CAN SAVE PATIENT'S VISIONS
}

\author{
Wan Mohd Aiman bin Wan Abdul Rahman', Nurull BahyaSuliman², Sujaya Sigh ${ }^{1 *}$ \\ ${ }^{1}$ Ophthalmology Department, Faculty of medicine, University Malaya, Malaysia \\ ${ }^{2}$ Ophthalmology Department, Hospital Tengku Ampuan Rahimah Klang, Malaysia \\ *Corresponding Author's Email: drsujaya@gmail.com
}

\begin{abstract}
Neovascular Glaucoma (NVG) is a severe form of secondary glaucoma characterized by proliferation of fibrovascular tissue in the anterior chamber angle. Clinically rubeosis iridis was seen at the pupillary margin and angle via gonioscopy.NVG results in guarded visual prognosis in view of aggressive clinical course and the difficulty in controlling the intraocular pressure(IOP).Patient treatment is indivdualized by taking into account patient visual prognosis, severity, level ofIOP, the cause of NVG, financial issues and patient's expectations. Trans-scleral cytophotocoagulations is one of the modalities available to reduce the intraocular pressure, usually reserved for the poor prognosis eye in view of its destructive nature. TSCPC in a good prognosis eye is always used in cautions.In this case series, we present 3 case of NVG whichwe use the early limited TSCPC to reduce the IOP to buy time for other managementsin which patient have good visual outcome.
\end{abstract}

\section{Keywords: TSCPC in NVG; Neovascular Glaucoma; TSCPC; CPC; Filtering Surgery}

\section{INTRODUCTION}

Neovascular Glaucoma is serious complication of ocular ischeamia often present as an end stage disease resulting blindness and pain(Fong, 2011 and Hossam et al, 2020). The treatment aim is to reduce the source of ischeamia such as pan retinal photocoagulations, anti- VEGF together with optimizing patient's comorbids such as diebetis mellitus and hypertension. Ocular therapy such as antiglaucoma eyedrops and systemic medications such as acetalzolamide included in the strategy to reduce the IOP. TSCPC is used to reduce the IOP thus reduce pain usually done in a poor prognosis eye. Surgical management such as trabeculectomy can reduce the IOP but it has higher failure rate in NVG. Thus glaucoma drainage device is more preferred in NVG unless patient has financial diffculties thus the former is performed. We present 3 cases of NVG where we give limited TSCPC (inferior 180 degree) in a good prognosis eye planning for filtering surgery.

\section{CASE STUDY}

Case 1

40 years old malay male with newly diagnosed diebetis mellitus presented in February 2018 to the eye clinic with sudden onset right eye (RE) blurring of visionand eye redness for the past two weeks.Patient went to the hospitaland was diagnosed with RE neovascular glaucoma(NVG) secondary to proliferative diebetic retinopathy and left eye advance eye disease .He was started with all four antiglaucoma and oral acetalzolamide. His eye wasnot in pain and his RE visual acuity was hand movement (HM). There's RE rubeosis iridis of 360 degree and despite all the antiglaucoma his intraocular pressure (IOP) is $48 \mathrm{mmHg}$. The cornea is clear and gonioscopy shows all four quadrant closed angle. Theres presence of nuclearoslerosis $1+$. His right eye fundus shows cup disc ratio of 0.4 , pre retinal heamorrhage at all four quadrant, new vessels at disc and resolving vitrous heamorrhage seen. In view of the uncontrolled IOP, he was given TSCPC inferiorly 180 degree $(25$ shots $x 1.5 \mathrm{mw} \times 1.5 \mathrm{sec})$. In a week the pressure falls to $42 \mathrm{mmHg}$. At the same time laser pan photocoagulation (PRP) was given until laser mark is adequate. In one month after the TSCPC, the IOP went down to 30 and patient undergo the triple procedure i.e RE phacoemulsification/intraocular lens implantation / and trabeculectomy with mitomycin $\mathrm{C}$ under local 
anesthesia. The surgery was uneventful. No antivascular endothelial growth factor (VEGF) was given prior to the surgery. Post operatively day 1 , the IOP falls down to $19 \mathrm{mmHg}$ and his vision improved to $6 / 36$. During the nine monthsfollow up, the bleb remains patent with IOP ranging $(6-12 \mathrm{mmHg})$ without antiglaucoma. His vision improved to $6 / 24$ with best corrected vision of $6 / 12$

\section{Case 2}

A 56 years old malay male with underlying diabetis mellitus for more than 10 years. He has done both eye cataract surgery in 2018. The RE was uneventful and has good vision of $6 / 6$. However his LE is complicated surgery where there'sposterior capsular rent.The IOL was inserted inside the bag but later noted to have sublaxated intraocular lens.Later he undergo LE IOL explantation and anterior chamber lens (ACIOL) impantation.His baseline pre operative vision LE is 6/18 and his post operativevision after ACIOL insertionwas 6/9.Before operation, his $\mathrm{BE}$ has no diabetic retinopathy with baseline cup disc ratio (CDR) of 0.4.However, eightmonth after operation, the patient developedRE severe non proliferative diebetic retinopathyand LE NVG secondary to proliferative diebetic retinopathy (PDR) where hepresented with LE redness and high IOP of $38 \mathrm{mmhg}$ and his vision drop to $6 / 60$.He was started on all 4 antiglaucoma and oral acetalzolamide. In the first 3 weeks the IOP falls down between range (24-28mmh). LE full PRP was also commenced as the cornea becomes clear. But his IOP raised again to 38 at 3 weeks and was given inferior TSCPC 180 degree ( 25 shots x $1.5 \mathrm{mwx}$ $1.5 \mathrm{sec}$ ). The IOP falls to $18 \mathrm{mmhg}$ three days later and oral acetalzolamide was stopped. After three weeks post TSCPC his IOP is raised again to $30 \mathrm{mmhg}$ but able to maintain between (14-26mmhg) with addition of oral acetalzolamide.Six weeks after TSCPC, he underwentuneventful LEtrabeculectomywith mitomycin $\mathrm{C}$ and his IOP falls down to $18 \mathrm{mmhg}$ without any antiglaucoma. No anti-VEGF was given prior to the surgery. During the next 8 month follow up, his IOP maintain ranging from $(4-12 \mathrm{mmhg})$ and his vision improved to $6 / 18$ with best corrected visionof $6 / 12$. His CDR LE increased from 0.4 to 0.6 .

\section{Case 3}

A 71 yearld Indian male, with underlying diabetis mellitus and hypertension has been under ophthalmology clinic follow up for his BE primary open angle glaucoma on two antiglaucoma (gutt timolast OM
BE and gutt xalatan ON BE). His baseline CDR is 0.9 both eyes. He also has no diabetic changes in both of his eyes. He also has underwent uneventful $\mathrm{BE}$ cataract surgery with BE post operative vision of $6 / 9$. However on october of 2019 he was diagnosed with LE NVG for investigation ?OIS/CRVOwhere he presented LE blurring of vision of perception of light all 4 quadrant, LE total hypheama with IOP $38 \mathrm{mmhg}$. This patient was on aspirin $75 \mathrm{mg}$ OD but no history of trauma to the eye. His right eye has no diabetic retinopathy or any hypertensive changes.B scan of the LE shows clear vitroues. Patient was admitted to the ward and was treated acutely with all 4 antiglaucoma and intravenous acetalzolamide together with topical steroids. Despite treatment given, the IOP still persistently high at $32 \mathrm{mmhg}$ and TSCPC was given at day 2 admission inferiorly 180 degree $(18$ shots x $1.5 \mathrm{mw}$ x $1.5 \mathrm{sec})$. After 5 days in ward the IOP falls to $20 \mathrm{mmhg}$ and the hypheama also subsides. The oral acetalzolamid was stopped. His vision improved to $6 / 36 \mathrm{ph} 6 / 24$. LE full PRP also commenced as the retina view is clear. The IOP is maintained below $20 \mathrm{mmhg}$ with 4 antiglaucoma until 3 weeks where the IOP increased back to $30 \mathrm{mmhg}$.Oral acetalzolamide was started again and the IOP able to maintain below 20mmhg. At 1 month post TSCPC, patient underwent uneventful LE trabeculectomy with mitomycin $\mathrm{C}$ and the IOP falls to $8 \mathrm{mmhg}$ without any antiglaucoma. No anti-VEGF was given prior to the surgery. During his nine month follow up, his LE IOP maintain ranging $8-10 \mathrm{mmhg}$ without any antiglaucoma and his LE best corrected vision is 6/9.

\section{DISSCUSSION}

Neovascular glaucoma is secondary glaucoma which difficult to manage. It involved reducing the source of ischemia,controlling the intraocular pressure, as well as inflammation. Once NVG was diagnosed a comprehensive management is warranted. For a patient with good visual prognosis, it is a race against time.

To reduce the source of ischemia, pan retinal photocoagulation (PRP) should be initiated as soon as theres a fundus view. A good adequate laser treatment can induce regression of the NVG. A short term treatment such as intravitreal anti-vascular endothelial growth factor such as ranibizumabcan also induce rapid regressionsof the new vessels(Moraczewski et al., 2009). In the case series however, no anti-VEGFwere given due to patients's financial constraints. The root cause of the ischeamicdrive such as diabetis mellitus should bemanaged properly. Prevention is better then 
cure, hence diabetic retinopathy screening play an important roleto detect early DR changes where patient should be comanage with the medical team to control their DM. Patient who is newly diagnosed with central retinal vein occlusion (CRVO) also should optimized their comorbids and tobe monitored closely for the new vessels at the angle.

Lowering the IOP as much as possible can prevent further damage of the optic nerve. Topical eyedrops such as postaglandin analogues, beta blocker, carbonic anhydrase inhibitors, and alpha adregenic agonist can be used. These medications will either reduce the the aqueous production or increase its outflow thus reducing the IOP. Topical administrations however usually insufficient and require further surgical intervention. Topical steroids is used to reduce the intraocular inflammation as well as cylopegics to reduce pain.

Cyclodestructive procedure such as TSCPC is an options used usually for poor prognostic eye. Theprocedure is hard to titrate the to attain desired IOP control. It can also induce more inflammation intraocularly and excessive treatment can lead to hypotony and phthisis bulbi. However there were increasing reports of its usage as an early alternative for treating NVG (Yildirim et al., 2009). As such in this case series, limited inferior 180 degree TSCPC can be useful and safe. Since the laser is not done fully for 360 degree, severe inflammation and hypotony could be avoided. The procedure was found to beuseful to lower down the IOP further andbuy some time before trabeculectomy with mitomycin $\mathrm{C}$ is done. It also presevered the conjuntiva superiorly where the bleb planned to be formed.

Trabeculectomy with mitomycin $\mathrm{C}$ were preferred in these cases as it is cheaper compare to GDD taking account patient's financial status. The success rate was reported up to $62.6 \%$ in 1 year and about $51 \%$ will decline in 5 years (Takihara et al., 2009). GDD is a good option as the failure rate is less dependant on the infammation compare to filtering bleb (Park et al., 2011 and, Noor et al., 2017).

\section{CONCLUSIONS}

NVG requires comprehensive treatment in controlling the IOP, retinal ischemia, and inflammation either medically or surgically. In this case series, the role of limited inferior TSCPC found to be useful and safe to lower down the IOP and is a potential consideration to be included in the treatment algorithm as an early intervention before proceeding to filtering surgery in a good prognosis eye.

\section{Conflict of Interest}

The author declares that they have no conflict of interest.

\section{ACKNOWLEDGEMENTS}

The authors are grateful to the patient for the information and descriptions.

\section{REFERENCES}

Fong, A.W., Lee, G.A., O'Rourke, P. \& Thomas, R. (2011). Management of neovascular glaucoma with transscleral cyclophotocoagulation with diode laser alone versus combination transscleral cyclophotocoagulation with diode laser and intravitreal bevacizumab. Clinical \& Experimental Ophthalmology, 39(4); pp:318-323.

Moharram, H.M., Abd-ElhamidMehanyElwan, S., Nassar, M.M. \& Abdelkader, M.F. (2020). Triple Procedure for Dense Cataractous Neovascular Glaucoma Patients. Journal of Ophthalmology, 2020.

Moraczewski, A.L., Lee, R.K., Palmberg, P.F., Rosenfeld, P.J. \& Feuer, W.J. (2009). Outcomes of treatment of neovascular glaucoma with intravitreal bevacizumab. British Journal of Ophthalmology, 93(5); pp:589-593.

Noor, N.A., Mustafa, S. \& Artini, W. (2017). Glaucoma drainage device implantation with adjunctive intravitreal bevacizumab in neovascular glaucoma: 3-year experience. Clinical Ophthalmology (Auckland, NZ), 11, pp:1417.

Park, U.C., Park, K.H., Kim, D.M. \& Yu, H.G. (2011). Ahmed glaucoma valve implantation for neovascular glaucoma after vitrectomy for proliferative diabetic retinopathy. Journal of Glaucoma, 20(7); pp:433438.

Takihara, Y., Inatani, M., Fukushima, M., Iwao, K., Iwao, M. \& Tanihara, H. (2009). Trabeculectomy with mitomycin $\mathrm{C}$ for neovascular glaucoma: prognostic factors for surgical failure. American Journal of Ophthalmology, 147(5); pp:912-918.

Yildirim, N., Yalvac, I.S., Sahin, A., Ozer, A. \& Bozca, T. (2009). A comparative study between diode laser cyclophotocoagulation and the Ahmed glaucoma valve implant in neovascular glaucoma: a longterm follow-up. Journal of Glaucoma, 18(3); pp:192-196. 